OAI-PMH: http://www.indteca.com/ojs/index.php/Revista Scientific/oai

Artículo Original / Original Article

\title{
Contexto de aprendizaje de estudiantes inmigrantes venezolanos de secundaria en el Perú (Estudios de casos)
}

\author{
Autor: Hernán Enrique Rueda Garcés \\ Universidad San Ignacio de Loyola, USIL \\ prof.ruedagarces@gmail.com \\ Lima, Perú \\ https://orcid.org/0000-0003-1334-6185
}

\section{Resumen}

El estudio se origina del interés en la inmigración registrada en el Perú; para el año 2020 ingresaron aproximadamente 860.000 venezolanos de forma legal, impactando el sistema educativo a nivel inicial y básico. A través de un abordaje interpretativo fenomenológico se estudiaron dos (2) casos de inmigrantes venezolanos, estudiantes de secundaria. El propósito de la investigación fue identificar los factores protectores y los factores amenazantes percibidos y atribuidos a los casos de estudio. Se identificaron como factores protectores: desempeño social de los inmigrantes venezolanos apoyado en las habilidades blandas; competencia comunicativa consolidada de los inmigrantes venezolanos, utilización del pensamiento divergente por parte de los inmigrantes venezolanos, entorno escolar amable y receptivo. En cuanto a los factores amenazantes dentro del contexto de aprendizaje: encuentro con una práctica docente que requiere valorar el pensamiento divergente, presencia de pensamiento orientado al etnocentrismo dentro del contexto de aprendizaje, excesiva valoración de los cursos del área de matemáticas y poca valoración a las áreas de lenguaje y sociales, percepción negativa de la venezolanidad por parte de algunos actores dentro del contexto de aprendizaje, necesidad de fomentar el trabajo colaborativo y baja implementación de la retroalimentación formativa dentro de la práctica docente.

Palabras clave: migración; contexto de aprendizaje; docencia.

Cómo citar este artículo:

Rueda, H. (2021). Contexto de aprendizaje de estudiantes inmigrantes venezolanos de secundaria en el Perú (Estudios de casos). Revista Scientific, 6(19), 142-163, e-ISSN: 2542-2987. Recuperado de: https://doi.org/10.29394/Scientific.issn.2542-2987.2021.6.19.7.142-163

Fecha de Recepción: 17-08-2020
Fecha de Aceptación: 18-12-2020
Fecha de Publicación: 05-02-2021 
OAI-PMH: http://www.indteca.com/ojs/index.php/Revista Scientific/oai

Artículo Original / Original Article

\title{
Learning Context of Venezuelan Immigrant High School Students in Peru (Case Studies)
}

\begin{abstract}
The study originates from the interest in immigration registered in Peru; By 2020, approximately 860,000 Venezuelans entered legally, impacting the educational system at the initial and basic level. Through a phenomenological interpretive approach, two (2) cases of Venezuelan immigrants, high school students, were studied. The purpose of the research was to identify the protective factors and the threatening factors perceived and attributed to the case studies. The following were identified as protective factors: social performance of Venezuelan immigrants supported by soft skills; Consolidated communicative competence of Venezuelan immigrants, use of divergent thinking by Venezuelan immigrants, friendly and receptive school environment. Regarding the threatening factors within the learning context: encounter with a teaching practice that requires valuing divergent thinking, presence of ethnocentric-oriented thinking within the learning context, excessive valuation of courses in the area of mathematics and little valuation of language and social areas, negative perception of Venezuela by some actors within the learning context, need to promote collaborative work and low implementation of formative feedback within the teaching practice.
\end{abstract}

Keywords: migration; learning context; teaching.

\footnotetext{
How to cite this article:

Rueda, H. (2021). Learning Context of Venezuelan Immigrant High School Students in Peru (Case Studies). Revista Scientific, 6(19), 142-163, e-ISSN: 2542-2987. Recovered from: https://doi.org/10.29394/Scientific.issn.2542-2987.2021.6.19.7.142-163
}

Date Received: 17-08-2020
Date Acceptance:

18-12-2020
Date Publication: 05-02-2021 


\section{Introducción}

El estudio sobre los movimientos migratorios humanos favorece la anticipación al impacto de este fenómeno sobre los involucrados, principalmente en el bienestar de los niños, niñas y jóvenes; destacando lo relativo a la educación. En el año 2016, se hace pública la Declaración de New York por parte de la Asamblea General de las Naciones Unidas (2016): donde se propone "asegurar que todos los niños estén estudiando en un plazo de unos meses después de su llegada" (pág. 8); de acuerdo al Global Compact for Migration (2018): la precitada propuesta de aseguramiento de estudios para todos los niños, se reitera en el proyecto de Pacto mundial para una Migración Segura (PPMMS); en este último documento, se enfatiza la necesidad de garantizar el acceso a la educación básica para los migrantes, reconociendo sus competencias y valorando el potencial beneficio del intercambio cultural.

Al indagar sobre la situación de recepción de migrantes en el Perú, de acuerdo con el informe DTM (2020): se confirma la información aportada por la Organización Internacional para las Migraciones (OIM) y el Fondo de las Naciones Unidas para la Infancia (UNICEF), según la cual para enero del año 2020, se registró el ingreso de aproximadamente 860.000 venezolanos de forma legal, cifra que pudiese ser mayor considerando el número de personas que ingresan sin pasar por los controles migratorios oficiales.

Considerando el alto impacto de la inmigración venezolana en el Perú, se presenta como propósito de la investigación identificar los factores protectores y los factores amenazantes percibidos y atribuidos en los estudiantes inmigrantes venezolanos en el Perú dentro el contexto de aprendizaje.

\section{Metodología}

A través de un abordaje fenomenológico, se estudian dos (2) casos de inmigrantes venezolanos en el Perú. En el estudio se implementaron 
entrevistas semiestructuradas a dos (2) jóvenes estudiantes venezolanos, a las madres de cada uno de los jóvenes estudiantes, a los profesores tutores de las aulas y a dos (2) compañero de aulas que fueron referidos como amistades, en total para este estudio se trabajó con ocho (8) entrevistas semi estructuradas. Las entrevistas se realizaron de forma presencial, entre los meses de julio a septiembre del año 2019, en la ciudad de Lima, Perú.

El primer caso se identificó con el nombre de Rubén: joven de 15 años, natural de la ciudad de Maracay, estado Aragua, quien cursó la totalidad de sus estudios en Venezuela en colegios privado hasta el $1^{\circ}$ de secundaria. En Perú ingresó en un colegio privado al $2^{\circ}$ de secundaria y al momento del recojo de la información estaba cursando el $3^{\circ}$ de secundaria.

El segundo caso se identificó con el nombre de Rosa: joven de 14 años, natural de Caracas, cursó estudios en Venezuela en colegios públicos hasta el $1^{\circ}$ de secundaria. En Perú ingresó a un colegio público al $2^{\circ}$ de secundaria, al momento del recojo de la información estaba cursando el $3^{\circ}$ de secundaria.

Se distinguieron dos tipos de información, las cuales se estructuraron como: a). autoreporte, cuando los informantes hacen referencia desde su propia experiencia; y b). la atribución externa, referida a la percepción sobre lo que puede estar experimentando otra persona desde la perspectiva del informante.

Los factores protectores fueron considerados como aquella categoría que hace referencia a las capacidades personales y a las características del contexto que facilite la incorporación y estadía en el ambiente escolar, ayude a elevar el rendimiento académico, favorezca la adaptabilidad social y promueve el ajuste emocional de los migrantes venezolanos en el contexto escolar en Perú. Los factores amenazantes serán considerados los que sean contrarios a los factores protectores. 


\section{Resultados}

\subsection{Caso 1. Rubén: Autoreporte de Factores Protectores}

Rubén identifica como factores protectores desde su experiencia en el contexto escolar como inmigrante venezolano en el Perú la capacidad personal para asumir con buen humor las situaciones donde hay discrepancias, actuando con asertividad y tolerancia a los comentarios y opiniones que pudiesen ser desencadenantes de controversia. Refiere que se apoya en el juego para evitar conflictos y reforzó el diálogo y el intercambio social.

Siguiendo con los factores protectores dentro del contexto escolar del informante identificado como Rubén; menciona la capacidad para exponer desde diferentes perspectivas los temas trabajados en clases. De acuerdo con Rubén, en temas de los cursos de plan lector, razonamiento verbal y ciencias sociales, informa que no requiere de mayor esfuerzo para dar respuestas que se orientan a exponer un punto de vista personal, en estas actividades marcó diferencias con el resto de sus compañeros de aula, en general sus opiniones eran diferentes a la mayoría del grupo, ya que los otros estudiantes mantenían un estilo de respuesta similar entre ellos, evidenciándose una tendencia entre el grupo a alinearse con un estilo de opiniones común.

El joven informante indica que el tener conocimientos sobre otras culturas le ha permitido tener otra visión de mundo; Rubén sugiere que tiene una visión más globalizada de la realidad, no solo centrada en el localismo y nacionalismo del Perú, considera que maneja una perspectiva cultural más amplia con conocimiento sobre Europa, Norteamérica y del mundo en general. Rubén valora como positivo la posibilidad de haber vivido en otro país, lo cual le ha permitido tener acceso a información variada.

Las características personales vinculadas con la creatividad y la proactividad, han favorecido formas diferentes de completar algunas tareas de clases, en ocasiones tomando la iniciativa; Rubén considera que esto le 
permitió aumentar la confianza en sí mismo y posicionarse dentro del salón de clases asumiendo cierto nivel de liderazgo en las actividades académicas. Finalmente, el apoyo y orientación de maestros y directivos, a través de un constante monitoreo y acompañamiento durante el proceso de adaptación; le reportó un mejor rendimiento escolar; principalmente por la orientación en actividades académicas que le eran nuevas o ajenas a su formación escolar previa.

\subsubsection{Atribución externa. Factores protectores: Madre de Rubén}

La madre de Rubén lo describe como un joven amigable y colaborador, estas dos características las asume la madre como un factor que le permite al joven adaptarse y ser recibido por nuevos grupos. Así mismo, lo considera un joven creativo y responsable en el cumplimiento de sus actividades; principalmente porque se siente satisfecho al cumplir con las tareas escolares. Para cumplir con las actividades escolares, propone formas novedosas de elaborarlas. Así mismo, el joven muestra un marcado interés por la lectura; lo cual le permite ser autónomo en la búsqueda de información y crítico al comparar diferentes documentos.

\subsubsection{Maestro tutor de Rubén}

El maestro de Rubén, lo describe como un joven respetuoso y obediente, indica que como alumno cumple con las normas del aula y con las tareas que le son encargadas. El tutor destaca que Rubén cuenta con facilidad para responder las preguntas que le hacen, así mismo por una alta capacidad oral para responder con fluidez y coherencia las preguntas en las sesiones de clases.

\subsubsection{Compañero de clases de Rubén}

El compañero de clases describe a Rubén como un joven alegre y 
bromista, siendo esto las principales características que valora como positivas, el compañero de clases afirma que Rubén es una persona que muestra una sonrisa en todo momento y se le ocurren bromas y chistes de forma constante. Se esfuerzo para cumplir con las actividades, se toma muy en serio las actividades y busca la solución de todo. Destaca la facilidad que el joven venezolano tiene para hablar en público de forma clara y con un nutrido léxico.

\subsubsection{Autoreporte de Factores Amenazantes}

Rubén identifica como factores amenazantes de su experiencia en el contexto escolar como inmigrante venezolano en el Perú: la percepción que tienen algunos compañeros sobre los venezolanos, especialmente en cuanto a los valores y conductas morales. Esta situación le causa incomodidad y le genera sentimientos de desconfianza hacia algunos compañeros o personas.

Por otro lado, percibe a los maestros como estrictos en cuanto al seguimiento de los patrones para las actividades y dan poco margen de espontaneidad para que los estudiantes puedan hacer las actividades de forma diferente; al saber que va a ser calificado negativamente hace que se ajuste a las exigencias de los docentes, dejando de lado los aportes que puede hacer a las actividades.

El desconocimiento de los eventos históricos o héroes del Perú, así como las tradiciones locales, le ha generado momentos incómodos; algunos compañeros o maestros no asumen que es un desconocimiento por su origen extranjero, lo califican como incapacidad intelectual, desinterés o en ocasiones irrespeto.

\subsubsection{Atribución externa. Factores amenazantes: Madre de Rubén}

La madre de Rubén enfatiza que él expresa constantemente que extraña su antiguo colegio y compañeros, principalmente por la camaradería y complicidad que existía con ellos. Conforme a lo expresado por la madre, 
Rubén valora a sus antiguos maestros como personas que fomentaban la creatividad y la participación. La madre del joven refiere que ha observado un proceso de disminución del interés en esforzarse en realizar algunas actividades porque ha identificado que existe un mínimo necesario de desempeño que satisface a los maestros.

Una de las principales amenazas que la madre de Rubén informa, es el desconocimiento del joven de la cultura, historia, tradiciones y valores patrios del Perú, por lo que ha recibido llamado de atención por no ajustarse a algunas conductas, como colocar el puño en el pecho al cantar el himno nacional.

\subsubsection{Maestro tutor de Rubén}

El principal factor amenazante que reporta el maestro tutor es la dificultad en seguir las directrices para realizar las actividades; el maestro indica que el joven implementa colores diferentes a los que se deben utilizar, tamaño o tipos de letras inusuales, realiza reportes o análisis distintos y en ocasiones utiliza formas de responder que no son las que se han enseñado en clases. También refiere, que el joven expone puntos de vista personales sobre temas que no son de análisis crítico sino de conocimiento, principalmente en el curso de Plan lector, donde se trabaja con evaluaciones objetivas sobre los textos y no análisis crítico de los mismos.

\subsubsection{Compañero de clases de Rubén}

El compañero de clases de Rubén, indica que el joven desconoce las directrices de ejecución de actividades de clases, así como las pautas a seguir para entregar las tareas y trabajos. Informa que constantemente Rubén cuestiona algunas opiniones de los maestros, o hace acotaciones que aparentan ser desagradables para los profesores; lo cual pareciera molestar a algunos docentes. Otro aspecto de resaltar son las dificultades en el área de matemáticas, principalmente lo atribuye a las pocas horas que dedica a la 
práctica y estudio de esa área, por lo cual es el curso que mayor dificultad le genera.

\subsection{Caso 2. Rosa: Autoreporte de Factores Protectores}

Rosa identifica como factores protectores de su experiencia en el contexto escolar como inmigrante venezolana en el Perú la facilidad personal para relacionarse con otros, Rosa expresa que ella busca establecer vínculos amistosos con sus pares, independientemente del género. Así como, un marcado interés en obtener nuevos conocimientos y busca información para resolver problemas y actividades, se apoya en otros compañeros que pueden tener consolidado el contenido a aprender.

Refiere tener conocimiento de la jerga local, lo considera como un factor protector en el nuevo contexto, ya que le facilita sus relaciones con los compañeros y profesores. Por otro lado, Rosa expresa que ha recibido apoyo por parte del personal que trabaja en la escuela. En general califica de positivo el ambiente escolar en cuanto a las actitudes de algunos maestros y personal que allí labora.

\subsubsection{Atribución externa. Factores protectores: Madre de Rosa}

La medre de Rosa informa que es una joven que realiza preguntas y comparte dudas con otras personas, no muestra temor o rechazo por informar cuando no conoce o se le dificulta algún contenido, buscando la explicación o apoyo necesario. Sustenta sus opiniones con base en fuentes de información diversas, se preocupa en indagar en diferentes contenidos para realizar actividades escolares, construyendo una visión amplia de los temas, esto le permite tener diferentes perspectivas; con toda la información que la joven maneja, fundamenta sus opiniones y juicios en las actividades escolares. 


\section{Artículo Original / Original Article}

\subsubsection{Maestro Tutor}

El maestro tutor reconoce en Rosa un excelente rendimiento con la entrega de las actividades y su participación en clases. Cumple con todas las actividades y tareas asignadas, es activa en las temáticas de clases, interviniendo y compartiendo conocimiento, toma la iniciativa para participar, reconoce en ella una alta capacidad comunicativa y de expresión oral.

\subsubsection{Compañera de clases}

La compañera de clases de Rosa la describe como una joven colaboradora, frecuentemente comparte sus tareas o apoya a los compañeros que tienen dudas; en general la caracteriza como una joven agradable y orientada a trabajar en grupo, define a Rosa como creativa y amable, conversa con todos, incluso con los que no conoce mucho, se le hace fácil conversar con otras personas.

\subsubsection{Autoreporte. Factores Amenazantes}

Rosa reconoce como factores amenazantes en el contexto educativo su desconocimiento de la expectativa de los maestros en cuanto los estilos de respuesta y modos de realizar las actividades, informa que le ha sido complicado ajustarse al modo en que valoran las actividades los profesores. El alto nivel de importancia que le asignan a las áreas numéricas y el poco valor que los maestros le dan a las áreas de ciencias sociales y literatura, ya que estas últimas son de su mayor interés.

Reconoce que desconoce y discrepa de algunos valores patrios del Perú, identifica diferencias importantes a los valores patrios de Venezuela, especialmente en lo referido a Simón Bolívar, ya que la imagen del Libertador de Venezuela en el Perú es negativa.

No se siente a gusto por el excesivo uso de las descalificaciones e incluso algunas amenazas con castigo físico para implementar la autoridad por 
parte de algunos maestros y personal del colegio. Indica que los profesores en su mayoría no orientan sobre las fallas de los alumnos en las actividades. Algunos maestros no explican la forma de hacer las correcciones o cuales son los modos adecuados de realizar las actividades.

$\mathrm{Ha}$ presenciado frecuentes descalificaciones hacia los venezolanos, Rosa reporta como algunos compañeros y algunos profesores han hecho comentarios negativos hacia los venezolanos, refiriendo comentarios peyorativos sobre las costumbres y calificándolos de desordenados, abusivos y faltos de respeto.

\subsubsection{Atribución de factores amenazantes: Madre de Rosa}

La madre de Rosa reporta que la joven le ha compartido expresiones de sentimientos negativos hacia profesores, ya que no toman en cuenta sus comentarios y opiniones, cuando no se alinean con los planteamientos del profesor. Esta situación le genera inseguridad, al igual que haber presenciado amenazas de algunas personas que trabajan en la institución con la utilización a posibles castigos físicos como medio disciplinario.

La madre manifiesta, que la joven le compartió el bajo nivel de confianza y de sinceridad entre los compañeros, percibe mucha competencia y uso de la descalificación entre compañeros de clases, ante lo cual se no se muestra de acuerdo.

\subsubsection{Maestro Tutor}

El maestro tutor refiere que Rosa no sigue en ocasiones las instrucciones para las actividades, el maestro explica que hay directivas específicas para cada actividad, tarea o evaluación; indica que Rosa en ocasiones presenta productos diferentes o no se ajusta a las indicaciones, por otro lado llega a conclusiones o interpretaciones diferentes a las que se solicitan, indica que es dispersa en algunas actividades, utiliza información 
diferente a la dada en las clases y en ocasiones ha contradicho lo que planea el maestro; el profesor enfatiza que debe cumplir con el objetivo de la clase y no abordar temas que no corresponden con el contenido del día.

\subsubsection{Compañera de Rosa}

La compañera de Rosa menciona, que en ocasiones la joven contradice los planteamientos de los maestros o hace críticas de las intervenciones de algunos alumnos y profesores. Los maestros le han indicado que debe respetar lo que se está presentando. Ante estas situaciones se muestra susceptible a los llamados de atención que le hacen los maestros; la compañera de clases relata que Rosa en ocasiones manifiesta tristeza o enojo ante algunas expresiones negativas de los maestros. Otro aspecto que destaca la informante es que Rosa ocasionalmente utiliza palabras extrañas que causan risa, esto le ha traído inconvenientes entre los compañeros, ya que algunos chicos lo han utilizado para hacer chistes desagradables.

\section{Integración de la información}

En el presente apartado, tomando como base la información recogida de los informantes para cada caso, se integran las descripciones resultantes de la síntesis derivada del proceso de análisis y categorización de la información; conforme a los contenidos pertinentes con las categorías de análisis que orientaron el estudio.

\subsection{Factores protectores de los inmigrantes venezolanos en el contexto escolar en el Perú}

a). Desempeño social apoyado en las habilidades blandas: de acuerdo con la información registrada y referida a los casos estudiados de migrantes venezolanos en el Perú, se identifica un adecuado desarrollo de las habilidades blandas. Al hablar de habilidades blandas o como otros autores 
denominan habilidades no cognitivas, se está haciendo referencia a lo que Ortega (2017): refiere como las competencias que tienen los individuos para establecer relación con otras personas, de forma empática, reconociendo, interpretando, respetando y actuando en sintonía con las emociones propias y ajenas; igualmente se considera la adecuada identificación de propósitos en sus acciones, asumiendo la responsabilidad que deriva de la toma de decisiones y afrontando situaciones conflictivas de manera asertiva, oportuna y adaptativa.

Las características asociadas con las habilidades blandas como factor protector de los inmigrantes venezolanos en Perú, con base a la información obtenida del autoreporte de los dos casos estudiados, así como por la atribución de características por parte de los maestros tutores, los familiares y los compañeros de estudio; hace referencia a la facilidad de establecer amistades a través de relaciones empáticas, fomentar el trabajo colaborativo y el compañerismo dentro del aula y fuera de la escuela. En la misma línea y considerando lo expresado por Heckman (2012): se ubica en estas habilidades llamadas blandas, asumir de forma positiva nuevas experiencias, actitud que forma parte del repertorio de comportamiento de los dos casos de venezolanos migrantes en el Perú, quienes identifican en su realidad la necesidad de afrontar el contexto de aprendizaje con la mejor disposición para avanzar con éxito.

b). Competencia comunicativa consolidada: en los casos estudiados y con base a la información recogida, se identifica como factor protector la competencia comunicativa. La competencia comunicativa, siguiendo lo expresado por Pasquali (1972): es entendida como la interacción entre individuos, donde se intercambian experiencias y se procura el consenso en las transacciones; del mismo modo que se forman vínculos y se definen objetivos comunes reconociéndose como iguales. La competencia comunicativa se identifica como un factor protector de los inmigrantes 
venezolanos en el Perú dentro del contexto de aprendizaje. Es de destacar que las actitudes, cualidades y formas comunicativas expresadas por los casos de estudio, han sido validadas como fortalezas en la adaptabilidad en el contexto de aprendizaje.

Desde una perspectiva individual y colectiva, tal como lo plantean Bermúdez y González (2011): la consolidación de la competencia comunicativa favorece el establecimiento de relaciones interpersonales productivas, mejora el clima organizacional y promueve la pertinencia a los grupos de referencia y pertenencia; esta afirmación está alineada con lo planteado por Hernández (2007): quien afirma que la competencia comunicativa requiere de comprensión y aceptación de las perspectivas de los otros, sin dejar de lado la perspectiva personal; validando la información que aporte cada persona, aceptando la diferencia de opiniones y teniendo control de las expresiones y emociones; características que han sido reconocidas como atributo de los casos en estudio.

c). Utilización del pensamiento divergente: un factor que fue identificado con base a la información recogida en el estudio, es la identificación de un estilo de pensamiento categorizado como divergente; el pensamiento divergente está asociado con la creatividad, principalmente si se toma como referencia lo dicho por Guilford (1970): quien propone que la creatividad se puede asumir como una capacidad cognitiva para procesar la información de forma fluida, asumiendo un enfoque flexible, buscando y aceptando lo original y novedoso de los abordajes con que se trabaja la realidad.

En el procesamiento de la información, se establecen asociaciones con otros temas o condiciones para que permitan tener una visión amplia para resolver problemas y generar nuevos conocimientos. En este sentido, en los inmigrantes venezolanos estudiados, se les atribuye estas características, en su comportamiento dentro de las escuelas.

d). Un entorno de aprendizaje amable: el entorno de aprendizaje en los 
casos de estudio, han sido contextualizados, presentan algunas barreras para la adaptabilidad de los migrantes venezolanos; sin embargo; ha facilitado la adecuada integración de los jóvenes extranjeros, principalmente por la consolidación de relaciones amistosas y participación en actividades grupales escolares; la mayoría de los maestros y personal docente ha sido valorado como receptivo y han apoyado la incorporación de los nuevos alumnos. Estas actitudes positivas facilitan los procesos adaptativos y permite generar un ambiente de seguridad.

\subsection{Factores amenazantes para los inmigrantes venezolanos en el contexto de aprendizaje en el Perú}

a). Encuentro con una práctica docente orientada al cumplimiento de criterios de ejecución y evaluación poco flexibles: para definir la práctica docente, se toma la propuesta de Vergara (2016): quien la precisa como las actuaciones resultantes de la interacción de todos los involucrados en el contexto de aprendizaje; siendo los principales actores los maestros, los directivos, los alumnos, los padres y madres, además de otros miembros de la comunidad; todos ellos vinculados con el proceso educativo.

Como resultado de las informaciones recogidas para este estudio, se identifica un modelo docente apegado a un conjunto de normas institucionales con poca flexibilidad para los involucrados, la presencia de criterios rígidos para la toma de decisiones y en general las formas de interacción entre maestros y alumnos se caracteriza por la baja afectividad. Adicionalmente se muestra un modelo disciplinario que se orienta a lo punitivo con poco espacio para el diálogo y el reconocimiento de las individualidades de los alumnos y el manejo de las emociones.

En cuanto a la importancia otorgada a las actividades académicas, por los involucrados en el entorno de aprendizaje, principalmente se valora el cumplimiento de las actividades como producto final, sin considerar el proceso 
o los aportes que puedan hacer los estudiantes. Se refuerza el cumplimiento de las directrices dictaminadas por los docentes o autoridades. Hay un desconocimiento de lo individual y del aporte personal de los estudiantes

b). Presencia de pensamiento orientado al etnocentrismo en el contexto de aprendizaje: considerando la información obtenida y el proceso de análisis, se identifican elementos en el contexto de aprendizaje relacionados con el pensamiento orientado al etnocentrismo, con base a lo expuesto por Cruz, Ortiz, Morocho y Orozco (2018): quienes lo entienden como "una doctrina ideológica o estilo del cual la persona observa al mundo de acuerdo con el contexto de su propia realidad" (pág. 180); entre otros aspectos el etnocentrismo se asocia con un sentimiento de superioridad cultural con respecto a otros grupos sociales.

Los casos informantes, así como las madres, refieren que los venezolanos inmigrantes en Perú han observado expresiones y juicios que orientan a la descalificación de otras culturas, costumbres, hábitos, tradiciones, uso del idioma, valores y personajes históricos. Estas formas de expresión se manifiestan tanto en algunos estudiantes como en docentes, lo cual dificulta la participación de los inmigrantes y ha generado sentimientos de desconfianza para compartir experiencias u opiniones que no están alineadas con las costumbres o valores del contexto.

c). Excesiva valoración de los cursos del área de matemáticas y poca valoración a otras áreas de formación: los maestros y autoridades escolares valoran el rendimiento en los cursos de matemáticas, álgebra y razonamiento numérico; lo cual orienta la formación hacia contenidos específicos de las áreas numéricas. Dando poca relevancia a cursos orientados a las letras $u$ otras ciencias. Desde esta perspectiva aparenta un desequilibrio entre las diferentes áreas de desempeño que un estudiante puede demostrar.

d). Percepción negativa de la venezolanidad: la matriz de opinión sobre los venezolanos ha generado en un identificable nivel de rechazo hacia el 
gentilicio y las personas de esta nacionalidad, lo cual puede tender al fenómeno de xenofobia entendida para Bouza (2002): como "[...] una de las formas más notables de discriminación [...] que se hace contra las personas ajenas al grupo nacional o étnico [...]" (pág. 4); este componente ha sido reconocido por los informantes dentro del contexto de aprendizaje, tanto por los jóvenes que son identificados como casos de estudio, así como para los otros informantes.

e). Bajo fomento del trabajo colaborativo: el trabajo colaborativo en el contexto de aprendizaje, en conformidad con Revelo-Sánchez, CollazosOrdóñez y Jiménez-Toledo (2018): representa un modelo educativo que fomenta la interacción entre los estudiantes para la construcción de saberes, conjugando las competencias individuales para alcanzar metas compartidas de forma consensuada. Tomando como base la información recogida, son pocas las actividades identificadas dentro y fuera del aula orientadas al trabajo colaborativo o a la generación de un ambiente académico que favorezca el apoyo espontáneo entre alumnos.

En el ambiente de aprendizaje estudiado, se caracteriza por favorecer los logros individuales y el reforzamiento de los éxitos personales sobre el trabajo en equipo. Este contexto puede intervenir como barrera para la aceptación y reconocimiento de desempeños que no se alineen a los estándares de ejecución, principalmente orientados a las individualidades; bajo este panorama los informantes refieren alto nivel de competitividad entre los estudiantes como una característica evidente.

f). Baja implementación de la retroalimentación formativa: en cuanto a la implementación de la retroalimentación formativa, el Currículo Nacional de Educación Básica del Perú, indica que se requiere que el estudiante tenga conocimientos de sus avances, acorde con las escalas valorativas alineadas con competencias claramente definidas, orientado a la mejora como resultado del desempeño logrado, en este sentido, el Ministerio de Educación (MINEDU, 
2016), indica que:

La retroalimentación consiste en devolver al estudiante información que describa sus logros o progresos en relación con los niveles esperados para cada competencia. Esta información le permite comparar lo que debió hacer y lo que intentó lograr con lo que efectivamente hizo. Además, debe basarse en criterios claros y compartidos, ofrecer modelos de trabajo o procedimientos para que el estudiante revise o corrija (pág. 180).

Los jóvenes venezolanos coinciden en informar que las orientaciones para superar fallas o mejorar rendimientos, no fue uno de los aspectos con los cuales los docentes apoyaron el periodo de adaptación al nuevo contexto educativo, así como en otras actividades donde la retroalimentación se limita a las calificaciones sin indicar los aspectos específicos a mejorar, espacialmente en las áreas numéricas.

Los maestros no aportaron en su justo momento las orientaciones pertinentes orientadas a la mejora, ni indagaron en las causas de rendimientos académicos por debajo de lo esperado; esta situación no favoreció la adaptación de los extranjeros al contexto educativo peruano.

\section{Conclusiones}

El abordaje de un fenómeno tan complejo como es la incorporación de población inmigrante al sistema educativo formal de un país receptor, puede tener infinidad de perspectivas, sin embargo, el estudio de casos desde un enfoque fenomenológico facilita aproximarse el impacto que tiene en los involucrados, desde una narrativa en primera persona.

En el estudio se presentaron dos casos con diferentes informantes, identificando los factores protectores y factores amenazantes en el contexto de aprendizaje de estudiantes inmigrantes venezolanos de nivel secundario en el Perú, destacando la inexistencia de una política de estado orientada a 
atender esta realidad.

Con base a la recogida de información, análisis y categorización de la información se concluye que los jóvenes estudiantes venezolanos tienen como uno de los principales factores protectores un desempeño social apoyado en las habilidades blandas, con marcada consolidación de las competencias comunicativas y expresión de la creatividad en las actividades académicas; estas características se alinean con un ambiente que se considera de forma general amable y receptivo.

Por otro lado, los factores que se identificaron como amenazantes para los inmigrantes venezolanos en el contexto educativo del Perú, destaca la práctica docente que no favorece la creatividad de los alumnos en las actividades académicas. En cuanto a la forma de exponer los contenidos académicos, se identifica una marcada orientación etnocéntrica, lo cual deja poco espacio de articulación a los valores, costumbre y tradiciones de los estudiantes extranjeros; se reporta la percepción negativa hacia la venezolanidad por parte de algunos integrantes de la comunidad educativa; adicionalmente, la práctica docente, no fomenta el trabajo colaborativo y con la retroalimentación formativa.

Finalmente, el contexto de aprendizaje es un ambiente complejo de interacciones y transacciones entre todas las personas que allí desempeñan un rol, siendo circunstancial toda acción que se pueda implementar para aumentar las probabilidades de éxitos de los inmigrantes venezolanos en el Perú. Cada acto que potencie las virtudes y capacidades de todos los estudiantes, docentes y personal que labore en la institución será valorada como una forma de eliminar las barreras que cada día aparecen en este contexto de aprendizaje.

\section{Referencias}

Bermúdez, L., \& González, L. (2011). La competencia comunicativa: 
elemento clave en las organizaciones. Quórum Académico, 8(1), 95110, e-ISSN: 1690-7582. Recuperado de:

https://www.redalyc.org/articulo.oa?id=199018964006

Bouza, F. (2002). Xenofobia. España: Glosario para una Sociedad Intercultural. Recuperado de: https://www.ucm.es/data/cont/docs/4712013-11-05-xeno3.pdf

Cruz, M., Ortiz, M., Morocho, F. y Orozco, P. (2018). Relativismo cultural, etnocentrismo e interculturalidad en la educación y la sociedad en general. ACADEMO. 5(2), 179-188. Recuperado de:

http://dx.doi.org/10.30545/academo.2018.jul-dic.10

DTM (2020). Monitoreo de flujo de la población venezolana en el Perú:

DTM Reporte 7. Perú: Organización Internacional para las Migraciones (OIM); Fondo de las Naciones Unidas para la Infancia (UNICEF).

Recuperado de: https://r4v.info/es/documents/download/74086

Global Compact for Migration (2018). Global Compact for safe, orderly and regular Migration. Morocco, North Africa: New York Declaration for Refugees and Migrants. Recovered from:

https://refugeesmigrants.un.org/sites/default/files/180713 agreed outc ome global compact for migration.pdf

Guilford, J. (1970). Creativity: Retrospect and Prospect. The Journal of Creative Behavior, 4(3), 149-168, ISSN: 0022-0175. Recovered from: https://doi.org/10.1002/j.2162-6057.1970.tb00856.x

Heckman, J. (2012). Hard evidence on soft skills. Focus, 29(2), 3-8, e-ISSN: 0195-5705. Recovered from:

http://www.irp.wisc.edu/publications/focus/pdfs/foc292b.pdf

Hernández, Y. (2007). La inteligencia y las competencias relacionadas.

Bogotá, Colombia: Editorial Los Libertadores.

MINEDU (2016). Currículo Nacional de la Educación Básica. Resolución

Ministerial Nro. 281-2016-ED. Lima, Perú: Ministerio de Educación. 
Recuperado de: http://www.minedu.gob.pe/curriculo/pdf/curriculonacional-de-la-educacion-basica.pdf

Naciones Unidas (2016). Declaración de Nueva York para los Refugiados

y los Migrantes. Nueva York, Estados Unidos: Asamblea General de las Naciones Unidas. Recuperado de:

http://www.un.org/es/comun/docs/?symbol=A/71/L.1

Ortega, C. (2017). Desarrollo de habilidades blandas desde edades tempranas. ISBN: 978-9942-960-18-4. Guayaquil, Ecuador: Centro de Publicaciones - Universidad ECOTEC.

Pasquali, A. (1972). Comunicación y cultura de masas. Caracas, Venezuela: Monte Ávila Editores.

Revelo-Sánchez, O., Collazos-Ordóñez, C., \& Jiménez-Toledo, J. (2018). EI trabajo colaborativo como estrategia didáctica para la enseñanza/aprendizaje de la programación: Una revisión sistemática de literatura. Tecnológicas, 21(41), 115-134, e-ISSN: 2256-5337. Recuperado de:

https://dialnet.unirioja.es/servlet/articulo?codigo $=6289046$

Vergara, M. (2016). La práctica docente. Un estudio desde los significados. Cumbres, 2(1), 73-99, e-ISSN: 1390-3365. Recuperado de: https://dialnet.unirioja.es/servlet/articulo?codigo $=6550779$ 


\section{Hernán Enrique Rueda Garcés \\ e-mail: prof.ruedagarces@gmail.com}

Nacido en Caracas, Venezuela, el 7 de febrero del año 1974.

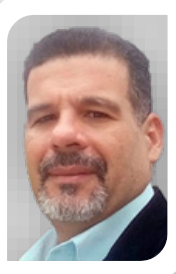

Doctor en Ciencias de la Educación por la Universidad Privada Dr. Rafael Belloso Chacín (URBE, 2010), Maracaibo, Venezuela; Licenciado en psicología en la Universidad Católica Andrés Bello (UCAB, 1998), Caracas; Licenciado en Psicología Educacional por la Pontificia Universidad Católica del Perú (PUCP, 2019), Lima, Perú; Magister Scientiarum en Gestión de Recursos Humanos por la Universidad Nacional Experimental "Rafael Marial Baralt" (UNERMB, 2007), Cabimas, Venezuela; Abogado por la Universidad de Los Andes (ULA, 2017), Mérida; docente universitario a nivel de pregrado desde el año 1999 y en postgrado desde el año 2007; actualmente docente de la Escuela de Postgrado en la Universidad San Ignacio de Loyola (USIL), Lima, Perú; Investigador académico y consultor en el área de investigación cualitativa; Ponente en eventos científicos internacionales desde el año 2009; Línea de investigación: Educación para inmigrantes.

El contenido de este manuscrito se difunde bajo una Licencia de Creative Commons ReconocimientoNoComercial-Compartirlgual 4.0 Internacional 